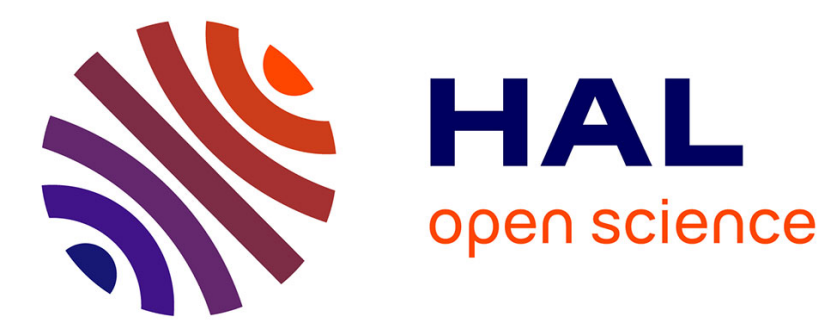

\title{
Can imperfect light polarization mimic parity violation in Stark experiments on forbidden Ml transitions?
}

\author{
M. A. Bouchiat, A. Coblentz, J. Guéna, L. Pottier
}

\section{To cite this version:}

M. A. Bouchiat, A. Coblentz, J. Guéna, L. Pottier. Can imperfect light polarization mimic parity violation in Stark experiments on forbidden Ml transitions?. Journal de Physique, 1981, 42 (7), pp.985-990. 10.1051/jphys:01981004207098500 . jpa-00209090

\section{HAL Id: jpa-00209090 https://hal.science/jpa-00209090}

Submitted on 1 Jan 1981

HAL is a multi-disciplinary open access archive for the deposit and dissemination of scientific research documents, whether they are published or not. The documents may come from teaching and research institutions in France or abroad, or from public or private research centers.
L'archive ouverte pluridisciplinaire HAL, est destinée au dépôt et à la diffusion de documents scientifiques de niveau recherche, publiés ou non, émanant des établissements d'enseignement et de recherche français ou étrangers, des laboratoires publics ou privés. 


\title{
Can imperfect light polarization mimic parity violation in Stark experiments on forbidden Ml transitions ?
}

\author{
M. A. Bouchiat, A. Coblentz, J. Guéna and L. Pottier \\ Laboratoire de Spectroscopie Hertzienne de l'E.N.S., 24, rue Lhomond, 75231 Paris Cedex 05, France
}

(Reçu le 24 février 1981, accepté le 25 mars 1981)

\begin{abstract}
Résumé. - Dans la discrimination du signal de violation de parité, un critère important est son caractère impair par renversement de la polarisation circulaire de la lumière excitatrice. Des imperfections du système de basculement de la polarisation ou une anisotropie dans les pièces d'optique peuvent engendrer des effets systématiques en dégradant la polarisation, faisant paraître partiellement impairs des signaux en principe pairs. Nous discutons l'intérêt d'un renvoi du faisceau sur lui-même dans la vapeur. Nous indiquons diverses méthodes pour minimiser les erreurs systématiques et pour contrôler leur valeur résiduelle, pendant l'expérience de violation de la parité, en utilisant les atomes eux-mêmes comme sonde.
\end{abstract}

\begin{abstract}
Oddness under reversal of the circular polarization of the exciting light is one important criterion to discriminate the parity violation signal. Imperfections of the polarization flipper or anisotropy in the optical components can generate systematic effects by spoiling the polarization and causing even signals to look partly odd. We discuss the interest of reflecting the beam back through the vapour. We indicate various methods to minimize systematic errors and to control their residual magnitude during the parity violation experiment while using the atoms themselves as a probe.
\end{abstract}

In an experimental search for a neutral-current induced right-left asymmetry in atoms [1], the most important difficulty is to discriminate the sought-for signal against the many possible spurious effects. Our experiment on the 6S-7S forbidden M1 transition of Cs in a d.c. electric field [2] looks for an upper-state electronic spin orientation which is odd under reversal of the electric field, or of the direction of the exciting beam, or of the sign of its circular polarization. Actually, this fourfold signature is fully specific only in principle, since the reversals are in practice imperfect to some extent. In a previous paper [3], we discussed the effect of improper field reversal; in the present paper, we examine the origins and consequences of improper polarization reversal.

1. Summary of the problem. - In our experiment, $\mathrm{Cs}$ atoms are exposed to a d.c. electric field $\mathbf{E}$ along $O y$ and excited by a circularly polarized laser beam tuned to the forbidden transition and directed along $\hat{\mathbf{k}} / / \mathrm{Oz}$ (Fig. 1). The electronic orientation $\mathbf{P}$ of the excited state is monitored in a direction $\hat{\mathbf{k}}_{\mathrm{f}}$ along $O x$. The parity violation $(\mathrm{PV})$ is predicted to appear as a small change of $\mathbf{P} . \hat{\mathbf{k}}_{\mathrm{f}}$ when the sign $\eta$ of the circular polarization of the laser beam is reversed. In addition, as a consequence of rotational invariance, under reversal

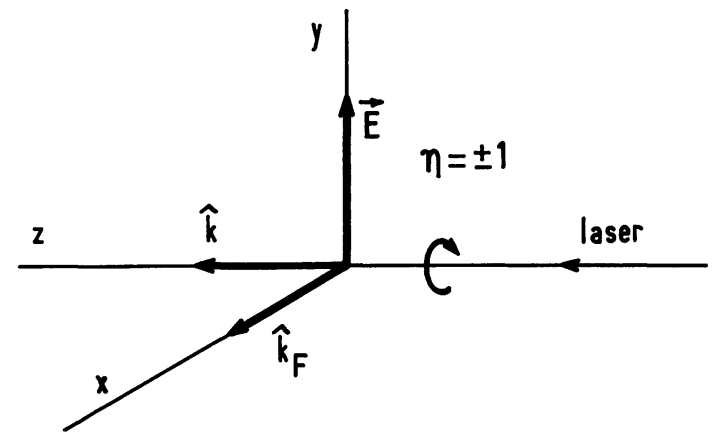

Fig. 1. - Scheme of the experimental configuration.

of $\mathbf{E}$ (equivalent to rotating the system half a turn around the beam) the $x$ (and $y$ ) components of the electronic orientation $\mathbf{P}$ are odd. Therefore the expected PV signal is odd under reversal of $\eta$ as well as of $\mathbf{E}$. In practice, reversing the sign $\eta$ of the circular polarization generally brings about a small change of the intensity and/or ellipticity as well, and consequently a small change of the parity conserving (PC) contribution to P. $\hat{\mathbf{k}}_{\mathrm{f}}$, i.e. a false asymmetry. Since the ratio of the PV to PC contributions is $\operatorname{Im} E_{1}^{\mathrm{PV}} / M_{1} \sim 10^{-4}$ for Cs (and $10^{-3}$ for T1 [4]), we shall derive below the intuitive result that an imperfection of that magnitude in the polarization flip (due 
e.g. to a stray birefringence $\left({ }^{1}\right)$ of $10^{-4}$ radian in a window) is liable to simulate the sought-for signal. We shall see in $\S 2.3$ that if the beam is reflected on a mirror and sent back through the atomic vapour, then the spurious effects associated with the forward and backward pass compensate each other while the PV signal is doubled. In practice, a reduction of at least two orders of magnitude can be achieved in this way, so that the imperfections in the optics can now reach the level of a few times $10^{-2}$ before they simulate the PV signal. However, a birefringence of $10^{-4}$ of the return mirror itself adds into the backward pass an imperfection which was absent in the forward one : therefore this imperfection is not compensated and is liable to simulate the expected PV signal. Thus, use of a double pass $\left({ }^{2}\right)$ attenuates by typically 2 orders of magnitude the requirements concerning all optical parts initially present on the single pass, but carries over the initial requirement onto the return mirror $\left({ }^{3}\right)$. This has to be taken seriously, since we have measured, on multidielectric mirrors, birefringences ranging from $2 \times 10^{-4} \mathrm{rad}$. to more than $10^{-3} \mathrm{rad}$. [5]. As long as less birefringent mirrors are not available, the solution seems to consist (i) in orientating the principal axes of the mirror birefringence in the direction which cancels the false asymmetry, and (ii) in combining results obtained with the birefringence axes interchanged by a $90^{\circ}$ rotation of the return mirror.

2. A more detailed analysis. - The fluorescence light emitted by the $\mathrm{Cs}$ atoms in the $7 \mathrm{~S}_{1 / 2} \rightarrow 6 \mathrm{P}_{1 / 2}$ decay is collected in the direction of observation $\mathbf{k}_{\mathrm{f}}$, which in principle coincides with $O x$ (Fig. 1); by means of a flipped (or modulated) $\sigma_{+}-\sigma_{-}$analyser, its circularly polarized intensity is measured. This yields directly the quantity

$$
\mathfrak{L}_{\mathrm{f}}=\operatorname{Tr} \rho \boldsymbol{\sigma} \cdot \hat{\mathbf{k}}_{\mathrm{f}},
$$

where $\rho$ is the density operator of the $7 \mathrm{~S}_{1 / 2}$ state and $\sigma$ is the set of Pauli matrices. In this quantity, the contribution that will cause trouble is the parity conserving one that is odd under reversal of the electric field; but for an irrelevant arbitrary normalization, its expression is, in the most general case [6] :

$$
\begin{aligned}
\mathcal{L}_{\mathrm{f}}^{\prime}=2 M_{1}\{\alpha \operatorname{Re}[ & \left.(\boldsymbol{\varepsilon} \cdot \mathbf{E})\left(\boldsymbol{\varepsilon}^{*} \times \hat{\mathbf{k}}\right)\right]+ \\
& \left.+g_{\mathrm{f}} \beta \operatorname{Re}\left[\boldsymbol{\varepsilon}^{*}(\boldsymbol{\varepsilon} \times \mathbf{E} \cdot \hat{\mathbf{k}})\right]\right\} \cdot \hat{\mathbf{k}}_{\mathrm{f}}
\end{aligned}
$$

(1) False asymmetries can originate in stray birefringences, but also in stray circular dichroisms. Since the latter appear experimentally to be generally smaller, we shall simply use the term « birefringence » throughout in this text.

$\left.{ }^{2}\right)$ This is true more generally for any even number of passes (as , in our experiment). The relevant quantity is then the average of the birefringence taken over all double-passes.

$\left({ }^{3}\right)$ As well as on the associated window, if the return mirror is located outside the cell as in the $\mathrm{Tl}$ experiment at Berkeley [4]. it has to be compared with the genuine PV contribution (written with the same normalization) :

$$
\mathfrak{L}_{\mathrm{PV}}=\operatorname{Im} E_{1}^{\mathrm{PV}}\left(\alpha+g_{\mathrm{f}} \beta\right) \operatorname{Im}\left(\boldsymbol{\varepsilon}^{*} \times \boldsymbol{\varepsilon} \cdot \hat{\mathbf{k}}\right) \cdot\left(\mathbf{E} \times \hat{\mathbf{k}} \cdot \hat{\mathbf{k}}_{\mathrm{f}}\right) .
$$

Here $\varepsilon$ is the complex polarization vector of the laser beam $; \hat{\mathbf{k}} / / O z$ is a unit vector along the beam; $M_{1}$ is the magnetic dipole transition amplitude; $i \operatorname{Im} E_{1}^{\mathrm{PV}}$ is the parity-violating electric dipole transition amplitude arising from weak neutral-currents; $\alpha E$ and $\beta E$ are the scalar and vector parts of the electric dipole transition amplitude induced by the electric field; $g_{\mathrm{F}}$ is the hyperfine $g$-factor of the upper-state. Written as above, equations (2) are valid for a $\Delta F=0$ transition; the corresponding expressions for $\Delta F= \pm 1$ transitions are given by equations (2) with the $\alpha$ terms omitted; therefore the following discussion applies to both cases. Equations (2) physically reflect the fact that the part that is linear in $\mathbf{E}$ in the polarized intensity $\operatorname{Tr} \rho \sigma$, originates in an interference between the mixed amplitude $M_{1}+i \eta \operatorname{Im} E_{1}^{\mathrm{PV}}$ and the fieldinduced amplitudes $\alpha E$ and $\beta E$.

Equations (2) clearly show that $\mathcal{L}_{\mathrm{f}}^{\prime}$ is reversed under reversal of the direction $\hat{\mathbf{k}}$ of the laser beam (with unchanged polarization $\varepsilon$ ) while $\mathcal{L}_{\mathrm{PV}}$ is not : therefore in a double-pass system, the false asymmetries associated with the forward and backward pass compensate each other - in so far as their polarization $\varepsilon$ is the same.

2.1 Simple-PASS CASE. - In the case of a simplepass along $O z$, with $\mathbf{E} / / O y$, equation $(2 a)$ reduces to :

$$
\begin{aligned}
\mathcal{L}_{\mathrm{f}}^{\prime}=2 E \cdot M_{1}\left\{\alpha\left|\varepsilon_{y}^{2}\right|\right. & +g_{\mathrm{f}} \beta\left|\varepsilon_{x}^{2}\right|- \\
& \left.-\left(\alpha-g_{\mathrm{f}} \beta\right) \chi_{y} \operatorname{Re} \varepsilon_{x}^{*} \varepsilon_{y}\right\},
\end{aligned}
$$

where $\chi_{y}=\hat{\mathbf{k}}_{\mathrm{f}} \cdot(\mathbf{E} /|\mathbf{E}|)$ characterizes the lack of orthogonality of $\hat{\mathbf{k}}_{\mathbf{f}}$ with $\mathbf{E}$. In the case of ideal $\sigma_{+}$ circular polarization, the quantities $\left|\varepsilon_{x}^{2}\right|,\left|\varepsilon_{y}^{2}\right|$ and $\operatorname{Re} \varepsilon_{x}^{*} \varepsilon_{y}$ are equal to $1 / 2,1 / 2$ and 0 respectively. In the real case, we take their imperfections into account by writing :

$$
\left.\begin{array}{rl}
\left|\varepsilon_{x}^{2}\right| & =(1 / 2)+\eta \lambda_{x} \\
\left|\varepsilon_{y}^{2}\right| & =(1 / 2)+\eta \lambda_{y} \\
\operatorname{Re} \varepsilon_{x}^{*} \varepsilon_{y} & =\eta \lambda_{\mathcal{R}} \\
\operatorname{Im} \varepsilon_{x}^{*} \varepsilon_{y} & =\eta / 2
\end{array}\right\}
$$

Here $\eta= \pm 1$ is the sign of the flipped circular polarization; the $\lambda$ 's are small independent real numbers (typically $10^{-3}$ to $10^{-1}$ in real instances). Possible small imperfections which do not reverse with $\eta$ are omitted, since they cannot simulate the sought-for signal. The intensity normalization $\varepsilon . \varepsilon^{*}$ is taken equal to 1 at the entrance of the polarization flipper. The spurious asymmetry arising from this 
improper circular polarization flip is then, according to equation (3) :

$$
\begin{aligned}
\mathcal{A}^{\prime} & =\mathcal{L}_{\mathrm{f}}^{\prime}(\eta=+1)-\mathfrak{L}_{\mathrm{f}}^{\prime}(\eta=-1)= \\
& =4 E M_{1}\left[\alpha \lambda_{y}+g_{\mathrm{f}} \beta \lambda_{x}-\left(\alpha-g_{\mathrm{f}} \beta\right) \chi_{y} \lambda_{\mathcal{R}}\right] .
\end{aligned}
$$

To eliminate the arbitrary normalization, we compare it with the true PV asymmetry, equal to

$$
\begin{aligned}
A^{\mathrm{PV}}=\mathcal{L}_{\mathrm{PV}}(\eta=+1)-\mathcal{L}_{\mathrm{PV}}(\eta & =-1)= \\
= & 2 \operatorname{Im} E_{1}^{\mathrm{PV}}\left(\alpha+g_{\mathrm{f}} \beta\right) E .
\end{aligned}
$$

The ratio of the false asymmetry to the true one is thus :

$$
\begin{aligned}
\mathcal{A}^{\prime} / \mathcal{A}^{\mathrm{PV}} & =2\left(M_{1} / \mathrm{Im} E^{\mathrm{PV}}\right) \times \\
\times & {\left[\alpha \lambda_{y}+g_{\mathrm{f}} \beta \lambda_{x}-\left(\alpha-g_{\mathrm{f}} \beta\right) \chi_{y} \lambda_{\mathcal{R}}\right] /\left(\alpha+g_{\mathrm{f}} \beta\right) . }
\end{aligned}
$$

In the case of the $6 \mathrm{~S}-7 \mathrm{~S}$ transition of $\mathrm{Cs}$, the ratio $M_{1} / \operatorname{Im} E_{1}^{\mathrm{PV}}$ is $\sim 10^{4}$, and $g_{\mathrm{f}} \beta / \alpha \sim-1.4 \times 10^{-2} \ll 1$. It is then clear that a defect $2 \lambda_{y} \sim 10^{-4}$ generates a spurious asymmetry equal to the genuine one, as we already announced. The defects $\lambda_{x}$ and $\lambda_{\mathcal{R}}$ are much less critical, due to the factors $g_{\mathrm{f}} \beta / \alpha$ and $\chi_{y} \ll 1$ (the misalignment angle $\chi_{y}$ can easily be kept of the order of a few times $10^{-2} \mathrm{rad}$.).

2.2 ORIGINS OF THE IMPERFECTIONS. - - The source of polarized light basically consists of a linearly polarized laser, followed by a « polarization flipper » $\left({ }^{4}\right)$, such as a $\lambda / 4$ plate or a Pockels cell whose axes are directed at $45^{\circ}$ from the incident polarization. Experimental checks on four different types of devices $\left(^{5}\right)$ evidenced for all four various imperfections : among others, the intensities, directions and linear polarization ratios of the transmitted beams corresponding to +1 and -1 circular polarization ratios are slightly different $\left({ }^{6}\right)$; these imperfections are well represented by equations (4) with typical values of the $\lambda$ 's ranging from $10^{-3}$ to $10^{-1}$.

- The polarization delivered by the flipper is then further spoiled by the anisotropic optical parts encountered by the beam before the atomic vapour. In the simple-pass case, this is simply the (generally birefringent) entrance window of the sample cell.

The action of a weakly anisotropic linear optical system on the complex polarization vector is represented in a quite general way by a complex $2 \times 2$

$\left({ }^{4}\right)$ Although we actually modulate continuously the polarization in our experiment, it is equivalent and simpler to discuss here in terms of flips.

$\left({ }^{5}\right)$ A quartz $5 \lambda / 4$ plate (Fichou, France); a Polaroid $\lambda / 4$ sheet; a transverse field Pockels cell (Lasermetrics $3030 \mathrm{HFW}$ ); a longitudinal field Pockels cell (Lasermetrics 1080 P).

$\left({ }^{6}\right)$ In the case of both Pockels cells, some of the imperfections critically depend on the orientation of the beam in the crystal. transfer matrix, which we expand on the basis of unity and Pauli matrices, in the form :

$$
\boldsymbol{\varepsilon}=\left[\left(\gamma_{0}+i \alpha_{0}\right) \mathfrak{1}+\sum_{k=1}^{3}\left(\gamma_{k}+i \alpha_{k}\right) \sigma_{k}\right] \boldsymbol{\varepsilon}^{\prime},
$$

or more explicitly :

$$
\boldsymbol{\varepsilon}=\left[\begin{array}{r}
1+\left(\gamma_{0}+\gamma_{3}\right)+i\left(\alpha_{0}+\alpha_{3}\right) \\
\left(\gamma_{1}-\alpha_{2}\right)+i\left(\alpha_{1}+\gamma_{2}\right) \\
\left(\gamma_{1}+\alpha_{2}\right)+i\left(\alpha_{1}-\gamma_{2}\right) \\
1+\left(\gamma_{0}-\gamma_{3}\right)+i\left(\alpha_{0}-\alpha_{3}\right)
\end{array}\right] \boldsymbol{\varepsilon}^{\prime}
$$

where the real coefficients $\alpha$ 's and $\gamma$ 's are all $\ll 1$. Physically, $\alpha_{0}$ and $\gamma_{0}$ respectively represent the (polarization independent) phase-shift and absorption; $\alpha_{3}$ is a birefringence of axes oriented along $O x$ and $O y$ and of optical phase-shift equal to $2 \alpha_{3} ; \alpha_{1}$ is a birefringence in the directions of the bisectrices of $O x$ and $O y$; simultaneous $\alpha_{1}$ and $\alpha_{3}$ produce a birefringence in a different direction; in a similar way, $\gamma_{1}$ and $\gamma_{3}$ correspond to linear dichroism ; $\alpha_{2}$ is an optical rotation, and $\gamma_{2}$ a circular dichroism.

Actually, if the optical system is inhomogeneous, the transfer matrix has to be treated as a random matrix. But as long as we limit our treatment to first order in the coefficients $\gamma_{k}, \alpha_{k}$, it can be shown that the description by equations ( 7 ) is still valid provided the average of the squared fluctuations affecting each anisotropy coefficient remains smaller than its average value, i.e. $\bar{\gamma}_{k}^{2} \ll \bar{\gamma}_{k}$ and $\bar{\alpha}_{k}^{2} \ll \bar{\alpha}_{k}$. Recent experimental results of ours [5] provide good indications that this condition is well fulfilled by our multipass cell.

According to equations (7), the defects $\lambda_{x}, \lambda_{y}, \lambda_{\mathcal{R}}$ of the polarization $\varepsilon$ seen by the atoms, are given in terms of the defects $\lambda_{x}^{\prime}, \lambda_{y}^{\prime}, \lambda_{\mathcal{R}}^{\prime}$ of $\varepsilon^{\prime}$ at the output of the flipper, by the following relations (treated to 1st order in the various defects $\alpha$ 's, $\gamma$ 's and $\lambda$ 's) :

$$
\left.\begin{array}{l}
\lambda_{x}=\lambda_{x}^{\prime}+\gamma_{2}-\alpha_{1} \\
\lambda_{y}=\lambda_{y}^{\prime}+\gamma_{2}+\alpha_{1} \\
\lambda_{\mathcal{R}}=\lambda_{\Re}^{\prime}+\alpha_{3}
\end{array}\right\}
$$

where the contributions from the flipper and from subsequent optics are clearly distinguished. Carrying these results into equation (6), we obtain :

$$
\begin{aligned}
& \mathcal{A}^{\prime} / \mathcal{A}^{\mathrm{PV}}=2\left(\alpha+g_{\mathrm{f}} \beta\right)^{-1}\left(M_{1} / \operatorname{Im} E_{1}^{\mathrm{PV}}\right) \times \\
& \quad \times\left\{\left[\alpha \lambda_{y}^{\prime}+g_{\mathrm{f}} \beta \lambda_{x}^{\prime}\right]+\left[\alpha\left(\gamma_{2}+\alpha_{1}\right)+g_{\mathrm{f}} \beta\left(\gamma_{2}-\alpha_{1}\right)\right]\right. \\
& \left.\quad-\left(\alpha-g_{\mathrm{f}} \beta\right) \chi_{y}\left[\lambda_{\mathcal{R}}^{\prime}+\alpha_{3}\right]\right\} .
\end{aligned}
$$

Note that among the 6 optical anisotropy parameters, only $\gamma_{2}$ (circular dichroism) and $\alpha_{1}, \alpha_{3}$ (birefringence) contribute. As expected from the naive argument of $\S 1$, the most harmful defects $\left(2 \lambda_{y}^{\prime}, 2 \alpha_{1}, 2 \gamma_{2}\right)$ can generate a false asymmetry equal to the genuine one as soon as they reach the level of $\left(\operatorname{Im} E_{1}^{\mathrm{PV}} / M_{1}\right) \sim 10^{-4}$. 
2.3 Double-PASS CASE. - According to equation (2) the spurious asymmetry associated with the forward pass in the direction $\hat{\mathbf{k}}$ is exactly compensated by the one associated with a backward pass of opposite direction $-\hat{\mathbf{k}}$, so that the net result is at first sight zero. The actual efficiency of this compensation is in fact limited by several causes :

i) First, even under quasi-normal incidence, real mirrors cause a slight change of the polarization [5]. We shall represent the effect of the return mirror by a transfer matrix similar to that of equation (7), but with roman coefficients $a$ 's and $c$ 's. Instead of zero, we now obtain the uncompensated effect of the return mirror, given by (compare with (9)) :

$$
\begin{aligned}
& \mathcal{A}^{\prime} / \mathcal{A}^{\mathrm{PV}}=\left(\alpha+g_{\mathrm{f}} \beta\right)^{-1}\left(M_{1} / \operatorname{Im} E_{1}^{\mathrm{PV}}\right) \times \\
& \quad \times\left[-\alpha\left(c_{2}+a_{1}\right)-g_{\mathrm{f}} \beta\left(c_{2}-a_{1}\right)+\left(\alpha-g_{\mathrm{f}} \beta\right) \chi_{y} \cdot a_{3}\right] .
\end{aligned}
$$

(The leading factor 2 of equation (9) has disappeared because the backward pass generates only one half of the total fluorescence intensity; the sign reversal originates in the reversal of the beam direction $\hat{\mathbf{k}}$.)

ii) A second limitation origins in the fact that the efficiencies of the forward and backward pass are most often different : this is due to the losses on the return mirror, and also, in the case where the paths of the two passes do not exactly coincide, to the fact that they are seen differently by the detector. As a result, the right side of equation (9) is no longer strictly compensated between forward and backward pass, but only reduced by some large "suppression coefficient » $S$, so that the ratio $\mathcal{A}^{\prime} / \mathcal{A}^{\mathrm{PV}}$ is so far given by :

$$
\mathcal{A}^{\prime} / \mathfrak{A}^{\mathrm{PV}}=\tilde{10}+(1 / \mathcal{S}) \cdot \tilde{9}
$$

where $\tilde{9}$ and $\tilde{10}$ denote the right sides of equations (9) and (10) respectively.

iii) In the case where the return beam makes a small but non-zero angle with the forward beam, a third limitation appears. Rigorously speaking, the equations written so far can no longer be applied just as they are, since they are written in a coordinate system bound to the beam. In fact, within terms of second order in this angle they remain unchanged, except the term containing $\chi_{y}$ in equation (9) : when the forward pass is replaced by the backward one, $\chi_{y}$ is modified in a way that is quite unpredictable in the absence of further assumptions; in the particular case where $\chi_{y}$ is exactly conserved, the $\chi_{y}$ term of equation (9) benefits from the suppression factor $\delta$ and equation (11) holds; but in the opposite particular case where $\chi_{y}$ is exactly reversed, the $\chi_{y}$ term in equation (9) is doubled in the double-pass instead of being suppressed. In the general case [6], the $\chi_{y}$ in equation (9) leads to some effective value $\tilde{\chi}$ for the double-pass, and the ratio $\mathcal{A}^{\prime} / \mathcal{A}^{\mathrm{PV}}$ is finally given by :

$$
\begin{aligned}
\mathcal{A}^{\prime} / \mathcal{A}^{\mathrm{PV}}= & \left(\alpha+g_{\mathrm{f}} \beta\right)^{-1}\left(M_{1} / \operatorname{Im} E_{1}^{\mathrm{PV}}\right)\left\{\left[-\alpha\left(c_{2}+a_{1}\right)-g_{\mathrm{f}} \beta\left(c_{2}-a_{1}\right)+\left(\alpha-g_{\mathrm{f}} \beta\right) \chi_{\mathrm{b}} \cdot a_{3}\right]+\right. \\
& \left.+(2 / \mathcal{S})\left[\alpha \lambda_{y}^{\prime}+g_{\mathrm{f}} \beta \lambda_{x}^{\prime}\right]+(2 / \mathcal{S})\left[\alpha\left(\gamma_{2}+\alpha_{1}\right)+g_{\mathrm{f}} \beta\left(\gamma_{2}-\alpha_{1}\right)\right]-2\left(\alpha-g_{\mathrm{f}} \beta\right) \tilde{\chi}\left[\lambda_{\mathcal{R}}^{\prime}+\alpha_{3}\right]\right\} .
\end{aligned}
$$

$\left(\chi_{\mathrm{b}}\right.$ is associated with the backward pass only, and is different from $\tilde{\chi}$, associated with both passes).

2.4 Multipass CASE. - In order to increase the signal in our experiment, the atomic vapour is placed between two mirrors fäcing each other, so that the laser beam performs a large number $N(\sim 50$ to 70$)$ of double-passes [7]. Equation (12) can be written for each double-pass with parameters $\chi_{\mathrm{b}, n},(1 / \$)_{n}, \alpha_{1, n}$, $\gamma_{2, n}, \tilde{\chi}_{1 n}, \alpha_{3, n}$ connected to this particular double-pass, and then averaged over the index $n$. The resulting expression will be identical with equation (12), except that the coefficients are now average values. In practice this brings two types of changes :

i) In addition to the anisotropy of the entrance window, the coefficients $\alpha_{1}, \alpha_{3}$ and $\gamma_{2}$ now contain the average anisotropy of previous double-passes, i.e. approximately $N / 2$ times the combined anisotropy of the mirror pair.

ii) From a detailed analysis [6], the final definition of $\tilde{\chi}$ turns out to be :

$$
\tilde{\chi}=(1 / s)\left\langle\chi_{y}\right\rangle+r . \delta \chi,
$$

where $\left\langle\chi_{y}\right\rangle$ is the average value of $\chi_{y}=\left(\hat{\mathbf{k}}_{\mathrm{f}}, \hat{\mathbf{y}}\right)$ over all passes, $\delta \chi$ is the standard deviation of the distribution of $\chi_{y}$ and $r$ is the correlation between $\chi_{y}$ and the forward or backward direction of the pass $(-1 \leqslant r \leqslant+1)$. In the extreme case $\chi_{y}=\left\langle\chi_{y}\right\rangle$, $\delta \chi=0$ (same $\chi_{y}$ for all passes), the suppression factor $1 / \delta$ is fully active, as was expected (cf. $\S 2.3$.iii); in the opposite extreme case $\left\langle\chi_{y}\right\rangle=0,|r|=1, \chi_{y}= \pm \delta \chi$ $\left(\chi_{y}\right.$ reverses exactly with the beam direction), equation (13) leads to $\tilde{\chi}=\left|\chi_{y}\right|$, i.e. there is no compensation at all, as was also expected. Equation (13) essentially says what happens in intermediate cases. Since in a practical situation $\left\langle\chi_{y}\right\rangle \lesssim \delta \chi$, the second term dominates and the suppression is uneffective. Therefore an experimental determination of $\tilde{\chi}$ is quite desirable. We shall present a simple method in $\S 3$.

3. Discussion. - We will now discuss the results, give orders of magnitude and outline methods to control and reduce the harmful terms.

The term that is by far the most dangerous one in equation (12) is the $a_{1}$ term, because there is no small 
factor in front to reduce it. This term says that a return mirror birefringence with axes at $45^{\circ}$ of the electric field transforms the $\sigma_{+}-\sigma_{-}$. flip into a change of the linear polarization along the field, without any compensation in the double-pass. Since $M_{1} / E_{1}^{\mathrm{PV}} \sim 10^{4}$ for the $6 \mathrm{~S}-7 \mathrm{~S}$ transition of $\mathrm{Cs}$, and since measured values of the birefringence $2 a_{1}$ are typically $5 \times 10^{-4} \mathrm{rad}$. [5], the corresponding spurious signal (SS) can easily reach 2.5 times the true signal (TS) ! As long as less birefringent mirrors are not available, a solution consists in orientating the birefringence axes of the return mirror parallel and perpendicular to the field : then $a_{1}$ is in principle zero; in practice, it will reasonably be reduced by at least one order of magnitude; this will bring it down to at most TS/4. It can then be further reduced by periodical $\pi / 2$ rotation of the return mirror $\left({ }^{7}\right)$, which reverses both $a_{1}$ and $a_{3}$ by interchanging the fast and slow axes (this also reduces the smaller $\chi_{\mathrm{b}} a_{3}$ term). In addition, according to our measurements [5], the circular dichroism $\left(2 c_{2}\right)$ is sufficiently small for its effect to be negligible $(\lesssim \mathrm{TS} / 100)$.

The next most dangerous terms are likely to be the ones that arise from the imperfections of the polarization flipper : experimental checks of four commercial devices $\left(^{5}\right)$ have given for $\lambda_{x}^{\prime}, \lambda_{y}^{\prime}$ and $\lambda_{\mathcal{R}}^{\prime}$ typical values of $10^{-2}$ and worst values of $10^{-1}$; combined with

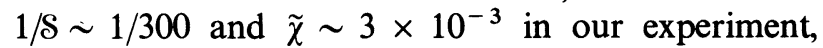
this leads to SS/TS $\sim 0.6$ (typically) to 6 (worst value) $\left(^{8}\right)$ ! We solve this difficulty by means of the improved polarization modulator which we describe in reference [5], the imperfections of which are 100 to 1000 times smaller.

The remaining terms contain the anisotropy of the entrance window and of the multipass. The effects of the birefringence $\left(\alpha_{1}, \alpha_{3}\right)$ as well as circular dichroism $\left(\gamma_{2}\right)$ are harmful : SS/TS $\lesssim 1$ or 2 ! Fortunately, these effects can be measured using the atoms themselves as a probe, and then compensated :

- the birefringence $\alpha_{3}$ (with axes parallel and perpendicular to the electric field) is able to transform a linear polarization $45^{\circ}$ off the field into a circular polarization. When a $\lambda / 4$ plate with axes parallel and perpendicular to the field is inserted after the polarization flipper [6], a non-zero $\alpha_{3}$ induces a small (flipped) circular polarization on the atoms which results in an easily detectable (flipped) atomic orientation along the laser beam $\left({ }^{9}\right)$. Once $\alpha_{3}$ is known, it

$\left({ }^{7}\right)$ From preliminary checks, the rotation of the mirror inside the Cs cell turns out to be quite realizable by means of a ferrofluidic rotary feedthrough.

$\left({ }^{8}\right)$ In the case of a Pockels cell, it is quite easy to compensate one given combination of $\lambda_{x}, \lambda_{y}$ and $\lambda_{R}$ by acting on the d.c. component of the driving voltage; but we find that the remaining uncompensated defects are still too large.

$\left({ }^{9}\right)$ A real plate will not be rigorously $\lambda / 4$. The error which would result can be eliminated by combining two measurements performed with the plate axes interchanged by a $\pi / 2$ rotation. can be compensated by a Bravais compensator. We expect a reduction by one or two orders of magnitude ;

- the birefringence $\alpha_{1}$ and the circular dichroism $\gamma_{2}$ transform the circular polarization flip into a small change of the linear polarization $\left|\varepsilon_{x}^{2}\right|-\left|\varepsilon_{y}^{2}\right|$ and of the intensity $\left|\varepsilon_{x}^{2}\right|+\left|\varepsilon_{y}^{2}\right|$ respectively. Thus both contribute to a small change of the total (unpolarized) fluorescence intensity. But for the small $\beta$ terms $\left(g_{\mathrm{F}} \beta / \alpha \sim-1.4 \times 10^{-2}\right.$ in our case), the Cs atoms are sensitive only to light polarized in the favoured direction of the electric field, so that the total fluorescence intensity depends only on $\left|\varepsilon_{y}^{2}\right|$; the change of this intensity depends only on $\lambda_{y}$, i.e. on the same combination $\gamma_{2}+\alpha_{1}$ (cf. Eq. (8b)) as appears in equation (12). As a result it can be shown that the spurious effect of $\gamma_{2}$ and $\alpha_{1}$ can be compensated by a change of the laser intensity performed so as to maintain the total fluorescence intensity constant when the circular polarization is reversed $\left({ }^{10}\right)$. In the real case of nonzero $\beta$, the compensation is not total but amounts to a reduction by a factor $\sim\left|g_{\mathrm{F}} \beta / \alpha\right| \sim 1 / 70$. A correct treatment of this problem is given in the Appendix.

Finally we would like to indicate how the parameters $\delta$ and $\tilde{\chi}$ can also be measured, using the atoms as a probe. The suppression coefficient $S$ is deduced from the comparison of the values of $\mathcal{L}_{\mathrm{f}}^{\prime}$ (see equations $(2 a)$ ) measured for a single-pass (which corresponds to $S=1$ ) and for the considered double- (or multi) pass; in such measurements, polarization defects can of course be neglected and the orientation signal is easily distinguished owing to its $E$-odd, $\eta$-even character. To measure the effective misorthogonality parameter $\tilde{\chi}$, we insert a quarterwave plate (with axes parallel and perpendicular to the field) : the flipped incident circular polarization is thus transformed into a flipped linear polarization at $45^{\circ}$ off the field; this produces a large known $\lambda_{\mathcal{R}}$. Consequently a large asymmetry $\mathcal{A}^{\prime}$ dominated by the last term in equation (12) must show up and its measurement leads to the determination of $\tilde{\chi}$.

Coming back to equation (12), we can now check that the various contributions can be identified and measured : the first one, by rotation of the return mirror; the second and third ones, by combining polarization defects observed in situ with measurements of the multipass suppression factor $\delta$ and effective misorthogonality $\tilde{\chi}$.

In conclusion, we have explicited in this paper various aspects of the risk of systematic error associated with polarization imperfections in a Stark PV experiment. In addition to increasing the counting rate, back reflection of the beam through the vapour

$\left({ }^{10}\right)$ In the case where c.w. lasers are used and where polarizations are modulated rather than flipped (as in our experiment), this intensity compensation is easily achieved with a servo loop. In the case where polarizations are actually flipped (as e.g. with pulsed lasers), it is simpler to divide the polarized fluorescence intensity by the unpolarized one. 
represents a good solution for minimizing this risk, provided the mirror is inserted inside the cell and with its anisotropy axes orientated parallel and perpendicular to $\mathbf{E}$. We have described various methods to still reduce the residual effects and we have shown that from the photocurrent of a single detector it is possible to extract in real time reliable and detailed information concerning the defects as they are seen in average by the excited atoms on which the PV test is performed.

Appendix. - We will now show that the spurious asymmetry arising from the $\gamma_{2}$ and $\alpha_{1}$ terms in equation (12) can be efficiently reduced by slightly changing the laser intensity so that the fluorescence intensity remains constant when the circular polarization of the laser is reversed.

The laser intensity change is accounted for by multiplying the right sides of equations (4) by a factor $(1+\eta \mu)$, where $\mu \ll 1$. Omitting higher-order terms as well as first-order terms even in $\eta$ (since they generate no false asymmetry), we obtain, in place of equations (4) :

$$
\begin{aligned}
\left|\varepsilon_{x}^{2}\right| & =(1 / 2)+\eta\left(\lambda_{x}+\mu / 2\right) \\
\left|\varepsilon_{y}^{2}\right| & =(1 / 2)+\eta\left(\lambda_{y}+\mu / 2\right) \\
\operatorname{Re} \varepsilon_{x}^{*} \varepsilon_{y} & =\eta \lambda_{\Re} \\
\operatorname{Im} \varepsilon_{x}^{*} \varepsilon_{y} & =\eta / 2
\end{aligned}
$$

Thus the only change is the replacement of $\lambda_{x}$ and $\lambda_{y}$ by $\lambda_{x}+\mu / 2$ and $\lambda_{y}+\mu / 2$ respectively. According to equations (8), this is equivalent to replacing $\lambda_{x}^{\prime}$ and $\lambda_{y}^{\prime}$ by $\lambda_{x}^{\prime}+\mu / 2$ and $\lambda_{y}^{\prime}+\mu / 2$ respectively. So, in the final result (12) we simply have to replace the quantity

$$
\begin{aligned}
& Q=\left(\alpha+g_{\mathrm{F}} \beta\right)^{-1}\left(M_{1} / \operatorname{Im} E_{1}^{\mathrm{PV}}\right)(2 / \mathrm{S}) \times \\
& \times\left\{\left[\alpha \lambda_{y}^{\prime}+g_{\mathrm{F}} \beta \lambda_{x}^{\prime}\right]+\left[\alpha\left(\gamma_{2}+\alpha_{1}\right)+g_{\mathrm{F}} \beta\left(\gamma_{2}-\alpha_{1}\right)\right]\right\}
\end{aligned}
$$

by :

$$
\begin{array}{r}
Q^{\prime}=Q+\left(\alpha+g_{\mathrm{F}} \beta\right)^{-1}\left(M_{1} / \operatorname{Im} E_{1}^{\mathrm{PV}}\right)(2 / \mathrm{S}) \times \\
\times\left(\alpha+g_{\mathrm{F}} \beta\right)(\mu / 2) .
\end{array}
$$

We must now express how $\mu$ is chosen. The total (unpolarized) fluorescence intensity is [6] :

$$
\mathcal{L} \propto\left|\varepsilon_{y}^{2}\right|+\frac{1+2 g_{\mathrm{F}}}{3} \frac{\beta^{2}}{\alpha^{2}}\left|\varepsilon_{x}^{2}\right|=\left|\varepsilon_{y}^{2}\right|+l\left|\varepsilon_{x}^{2}\right|,
$$

where $l \# 5 \times 10^{-3} \ll 1$ in our case. According to equations (8) and (14), the spurious asymmetry of $\mathcal{L}$ is :

$\mathfrak{L}(\eta=1)-\mathfrak{L}(\eta=-1) \propto 2 \lambda_{y}+2 l \lambda_{x}+(1+l) \mu$.

We choose $\mu$ so as to cancel this quantity, i.e. (cf. Eqs. (8)) :

$$
\mu=-2\left[\frac{\left(\lambda_{y}^{\prime}+l \lambda_{x}^{\prime}\right)}{1+l}+\gamma_{2}+\frac{1-l}{1+l} \alpha_{1}\right] .
$$

Carrying this into equations (15) we find, to first-order in the small quantities $l$ and $m=g_{\mathrm{F}} \beta / \alpha \propto-1.4 \times 10^{-2}$, that the spurious asymmetry $Q$ is reduced to :

$$
Q^{\prime}=\left(M_{1} / \operatorname{Im} E_{1}^{\mathrm{PV}}\right)(2 / \delta)(l-m)\left[\left(\lambda_{y}^{\prime}-\lambda_{x}^{\prime}\right)+2 \alpha_{1}\right] .
$$

Thus the effect of the circular dichroism $\gamma_{2}$ is totally compensated, and those of the birefringence $\alpha_{1}$ and of the flipper imperfections $\lambda_{y}^{\prime}$ and $\lambda_{x}^{\prime}$ are reduced by a factor $|l-m| \sim 10^{-2}$. (They would be totally compensated if $\beta$ were zero.)

\section{References}

[1] For a review about neutral-currents and their effects in Atomic Physics, see e.g. Bouchiat, C., J. Phys. G (Nucl. Phys.) 3 (1977) 183 and references quoted there.

[2] Bouchiat, M. A. and Pottier, L., Phys. Lett. 62 (1976) 327; Springer Series in Optical Sciences, vol. 7 : Laser Spectroscopy III, edited by J. L. Hall and J. L. Carlsten (1977), p. 9; see also Proc. of the Workshop on Neutral Current Interactions in Atoms, Cargèse (1979), ed. by W. Williams, Univ. of Michigan, Ann Arbor, Mich. (USA).
[3] Bouchiat, M. A., GuÉna, J. and Pottier, L., J. Physique Lett. 41 (1980) L-299.

[4] Conti, R., Bucksbaum, P., Chu, S., Commins, E. and HunTER, L., Phys. Rev. Lett. 42 (1979) 343.

[5] Bouchiat, M. A. and PotTiER, L., to appear in Opt. Commun.

[6] GuÉnA, J., Thèse de $3^{\mathrm{e}}$ cycle, Université Paris VI (24.2.81), unpublished.

[7] Bouchiat, M. A., Pottier, L. and Trénec, G., Revue Phys. Appl. 15 (1980) 785. 